\title{
Estilos cognitivos y percepción de la función familiar en estudiantes de básica primaria ${ }^{1}$
}

\author{
Luz Elena Piedrahita Gómez ${ }^{2}$, Elisabeth Suaza Patiño ${ }^{3}$ \\ NotA $^{4}$
}

\section{Resumen}

Este artículo sintetiza los resultados de una investigación, llevada a cabo con el objetivo de caracterizar los estilos cognitivos en la dimensión dependencia e independencia de campo y la percepción de la función familiar en estudiantes de básica primaria de una institución educativa del sector oficial, teniendo como principal prioridad que la escuela, por obvias razones, tiene la mayor responsabilidad en la educación de los niños y las niñas en edad escolar, pero no es la única que incide en este aspecto trascendental de la vida de los seres humanos, pues, como han evidenciado muchos estudios, la familia juega un papel fundamental en esta tarea.

Los resultados del estudio, confirman hallazgos de otras investigaciones en cuanto a la relación entre los estilos cognitivos, la edad y el grado escolar. La funcionalidad familiar es percibida por el $66 \%$ de los estudiantes como disfuncional leve, en razón de lo cual, es necesario continuar profundizando en el tema, con el fin de que la sociedad en su conjunto y la escuela en particular, puedan articular mejor su accionar con la familia, para obtener los resultados deseados.

Palabras clave: Estilos cognitivos, dependencia e independencia de campo, percepción función familiar, disfuncionalidad familiar leve.

\section{Cognitive Styles and Perception of Family Basic Function in Elementary Students}

\begin{abstract}
This article summarizes the results of research, developed with the aim of determining the relationship between perceptions of family function and cognitive styles of students from elementary school, with the school principal priority, for obvious reasons, has the greatest responsibility in the education of children of school age, but is not the only incident in this crucial aspect of human beings, for as many studies have shown, the family plays a key role in this task.
\end{abstract}

1 Recibido: 05 de mayo de 2016. Aceptado: 28 de octubre de 2016.

2 Luz Elena Piedrahita Gómez. Magíster en Educación Docencia; Enfermera universidad de Caldas. Correo electrónico: luziernaga63@hotmail.com

3 Elisabeth Suaza Patiño. Magíster en Educación Docencia; Enfermera, Universidad Libre de Pereira. Correo electrónico: lizsuaza@hotmail.com

4 Trabajo asesorado por María Inés Menjura Escobar. Doctora en Ciencias Sociales, Niñez y Juventud. Docente asociada, Facultad de Ciencias Sociales y Humanas. Universidad de Manizales. Correo electrónico: mime@umanizales.edu.co 
The study results confirm findings of other studies regarding the relationship between cognitive styles, age and grade. Family functioning is perceived by $66 \%$ of students as mild dysfunctional, because of which, it is necessary to continue to deepen the subject, so that society as a whole and the particular school can articulate better their operate with the family, to get the desired results.

Keywords: Cognitive styles, dependence and field independence, perception family function, mild family dysfunction.

\section{Presentación}

Los avances en la investigación y los requerimientos de la sociedad actual demandan de la escuela conocer los procesos de aprendizaje de los estudiantes para adoptar nuevas formas de enseñanza. La escuela, como centro incuestionable de formación, debe conocer las particularidades de sus estudiantes; ya no se puede pensar en estudiantes promedio, porque cada uno, investido de su propia individualidad reclama un tipo de intervención específica que la institución educativa debe atender, so pena de poner en tela de juicio su papel misional. Una de estas características es la relacionada con los estilos cognitivos de los estudiantes, sobre todo en los primeros años de aprendizaje, para realizar las adecuaciones e intervenciones pertinentes.

Los estilos cognitivos son definidos por Witkin (1976, p. 39) como los "modos característicos de funcionar que revelamos a través de nuestras actividades perceptivas o intelectuales de una manera altamente estable y profunda", expresan sintéticamente que las personas aprendemos de manera distinta, y que a la vez, existen una serie de dimensiones que intervienen en nuestra manera de ser, pensar y actuar.

El estudio de los estilos cognitivos ha tenido mucho auge en las últimas décadas en el ámbito latinoamericano, como se evidencia en los innumerables estudios realizados sobre el tema. En particular, los estudios relacionados con los estilos cognitivos y la interacción familiar, han destacado la influencia del contexto familiar sobre los procesos cognitivo-motiva- cionales del estudiante y el aprendizaje en situaciones escolares (Mori, 2002).

En el ámbito familiar el sujeto desarroIla las primeras percepciones acerca de sí mismo y en la edad escolar, la familia ejerce un significativo impacto sobre el aprendizaje y el rendimiento académico. De acuerdo con ello, la actitud y la conducta de los padres, así como sus creencias y expectativas con relación a los logros de sus hijos favorecen el desarrollo de un auto concepto académico positivo o negativo e influyen en la naturaleza de sus motivaciones (extrínseca, intrínseca, bajo o alto nivel de logro, etc.)

En este sentido, la investigación que deriva el artículo, buscó caracterizar los estilos cognitivos en la dimensión dependencia/independencia de campo y la percepción de la función familiar de niños y niñas de grados $1^{\circ}$ a $3^{\circ}$ de una Institución Educativa Oficial del Municipio de Dosquebradas, -Risaralda-, Colombia.

\section{Justificación ${ }^{5}$}

Las personas manifiestan ciertas particularidades respecto a la forma como perciben, procesan y utilizan la información, hecho claramente comprobado por diversas investigaciones, lo que hace que sea de interés para los educadores y demás actores involucrados en el proceso educativo. Estas particularidades son

$5 \quad$ Este artículo se deriva de la investigación titulada Estilos Cognitivos y Percepción de la Función Familiar en Estudiantes de Básica Primaria, realizada en el período comprendido entre 20132014 , 
conocidas como estilos cognitivos. Una de las dimensiones más estudiadas es la independencia/dependencia de campo. La primera de ellas, consiste en la capacidad de aislar un determinado rasgo que se percibe como relevante del contexto en el que se integra y la segunda-dependencia de campo- se caracteriza por la tendencia a percibir un fenómeno como un todo unitario, sin atender a las diferentes partes que lo integran.

La configuración de los estilos cognitivos puede obedecer a muchos factores o ciertos modos de caracterización de las formas de percibir, recordar y pensar, o a maneras distintas de descubrir, almacenar, transformar y utilizar la información y corresponde a los investigadores indagar las diferentes relaciones que puedan existir entre cada uno de esos factores, respecto al estilo cognitivo.

Conocer las diferencias en los estilos cognitivos de los estudiantes puede ser de gran ayuda, ya que permite comprender los modos o formas de funcionamiento intelectual más apropiadas, las habilidades más características, las estrategias de aprendizaje preferidas, así como las actitudes de los estudiantes. Por lo tanto, considerar las diferencias individuales de los estudiantes a través de sus estilos cognitivos, es necesario para programar, seleccionar las metodologías, evaluar y por supuesto, orientar escolar y profesionalmente, a los estudiantes (García, 1989).

Identificar el estilo característico de cada estudiante debe ser un propósito de los educadores, hecho muchas veces ignorado, especialmente si se tiene en cuenta que estas particularidades son variables en los niños y en las niñas interviniendo varios factores, entre ellos, la percepción del funcionamiento familiar. Este argumento tiene mayor validez, si se tienen en cuenta algunos resultados de investigaciones, entre las que se destaca la realizada por Páramo y Tinajero (1992)

quienes concluyen que los ambientes familiares que permiten al niño organi- zación y estructuración de sus propias experiencias favorecen el desarrollo de un estilo cognitivo más independiente de campo.

Estudios de esta naturaleza son útiles a educadores y padres de familia, para conocer mejor su papel y su responsabilidad en la educación de los niños y las niñas. Las investigaciones demuestran, que los estilos cognitivos en la dimensión dependencia-independencia de campo, presentan determinadas características que favorecen los procesos de aprendizaje, relacionadas con la capacidad para resolver problemas y llevar a cabo tareas sin necesidad de instrucciones, analizar una situación y reestructurarla, poseer iniciativa y utilizar sus propios criterios para desarrollar tareas o actividades, así como tolerar las críticas y el fracaso, entre otras condiciones.

Por su parte, el funcionamiento familiar influye en el desarrollo socioemocional y en el desempeño académico de los hijos de acuerdo con los estudios realizados por Avanzini (1995); Valdés, Esquivel y Artiles (2007), lo cual puede explicarse por los efectos negativos que se derivan de un mal funcionamiento familiar en la participación efectiva de los padres en la educación de los hijos, en aspectos relacionados con las pautas de crianza, el seguimiento del aprendizaje en la casa y la comunicación con la escuela, aspectos que pueden ser limitados cuando la familia no presenta un funcionamiento adecuado (Valdés, Ángel y Urías, 2010).

En coherencia con los planteamientos anteriores, una aproximación al estudio de los estilos cognitivos y a la percepción del funcionamiento escolar de estudiantes de básica, provee a los docentes conocimientos sobre las diferencias en el aprendizaje y por consiguiente la adopción de estilos de enseñanza que respondan a las diferencias individuales y al emprendimiento de acciones con las familias para su vinculación efectiva en los procesos de aprendizaje de sus hijos. 


\section{Antecedentes}

Las investigaciones realizadas sobre los estilos cognitivos en la dimensión dependencia-independencia de campo (DIC), en el ámbito internacional como nacional son numerosas, pero han sido escasos los estudios que exploran los estilos cognitivos y la influencia del contexto familiar.

En Colombia, los estudios realizados por el grupo de investigación liderado por Hederich, a partir de la década de los años 90 , ha permitido consolidar un programa de investigaciones. Las líneas que han tenido mayor desarrollo en el grupo son: Estilo cognitivo y Logro Educativo, Efectividad Diferencial de Modelos Educativos y Pedagógicos, Estilos Cognitivos de Docentes y Estudiantes y Estilo Cognitivo y Movilidad Funcional.

El estudio sobre "Regiones cognitivas de Colombia", liderado por Hederich, Camargo, Guzmán y Pacheco (1995), se llevó a cabo con el propósito fundamental de demostrar la existencia de diferencias cognitivas entre individuos colombianos, pertenecientes a distintas tradiciones culturales regionales, aportando la subregionalización de algunas zonas que mostraban cambios recientes, entre ellas, las Zonas del Pacífico, dividido en zonas costeras y partes del valles del río Cauca y el complejo del valle del Magdalena, comprendido en zona alta y zona media. La mayor parte de la zona pacífica y Magdalena tienen gran concentración de población afrodescendiente.

El resultado de este trabajo es la hipótesis del individualismo-colectivismo cultural, debido a que, según Hederich (2001) el proceso de mezcla racial, curiosamente no se dio en esta región y, como consecuencia, los grupos afro de esta zona, desarrollaron una sociedad bastante aislada del resto del país. Por lo tanto, en esta región se conservan, aún visibles, las tradiciones africanas.

La investigación titulada "Estilo cognitivo en la dimensión independencia- dependencia de campo (DIC), influencias culturales e implicaciones para la educación" realizada por Hederich (2004),recoge la trayectoria de los estudios realizados durante quince años sobre el tema. El estudio comprende tres partes. En la primera parte, el autor aporta bases para la conceptualización de los estilos cognitivos en la dimensión dependencia e independencia de campo, en la perspectiva histórica, actual y las proyecciones futuras.

En la segunda parte, describe la relación entre la cultura y los estilos cognitivos (la mayor independencia o la mayor sensibilidad al medio-dependencia-), basada en indicadores de conformidad social como: la estructura social rígida, la relación con el énfasis en el seguimiento a la autoridad civil o religiosa, el egocentrismo - sociocentrismo, y el patriarcalismo - matriarcalismo familiar. Hipótesis cuyos resultados permitieron mostrar que las influencias culturales se manifiestan de forma clara en el estilo cognitivo en la dimensión independencia - dependencia de campo, de las personas que conforman los complejos culturales colombianos.

Así en la Región andina y santandereana fue posible identificar mayor tendencia a la independencia de campo, en oposición a la Región Costera del Litoral del Pacífico. El estudio, concluye la presencia de influencias culturales claras en la dimensión independencia-dependencia de campo, que pueden ser explicadas por la estructura familiar -patriarcal o matriarcal- y, por la orientación egocéntrica o socio-céntrica del grupo.

La tercera parte de la investigación, realizada por Hederich (2004), da cuenta de las implicaciones educativas de los estilos cognitivos. En esta perspectiva, el estudio llevado a cabo por el autor, con un grupo de estudiantes de $8^{\circ}$ y $10^{\circ}$ grados de instituciones educativas del sector oficial de Bogotá, con el propósito de estudiar la relación entre los estilos cognitivos y los diferentes indicadores de logro educativo, entre otros aspectos, incluidos los logros 
obtenidos a través de pruebas objetivas y los reportes de las evaluaciones pedagógicas de los maestros, señala que los estudiantes que mostraban mejores logros en las pruebas (la mayoría con un comportamiento rebelde) tendían hacia la independencia de campo, mientras que los grupos con un desempeño bajo (la mayoría con buena disciplina) tendían hacia la sensibilidad al medio.

El estudio concluye señalando que el sistema educativo colombiano, es paradójico por cuanto al intentar formar a los estudiantes en, y para la independencia del medio, facilita el logro de aprendizajes para este tipo de estudiantes y excluye a aquellos estudiantes que por su sensibilidad al medio, no aprenden ni logran las competencias mínimas.

La investigación realizada por Loscos (2001), sobre "Autorregulación del estilo cognitivo a través del lenguaje", plantea que las características más significativas de la dimensión dependencia e independencia de campo (D.I.C), es su adaptabilidad al medio, la cual puede desarrollarse con un entrenamiento específico, mediante un aprendizaje perceptivo.

Las conclusiones del estudio señalan, que el estilo cognitivo, en la dimensión dependencia-independencia de campo, es una forma de procesar la información perceptiva y destaca la función mediadora del lenguaje sobre la percepción. Después de realizar el proceso de intervención, se puede concluir que el estilo cognitivo es modificable o, más específicamente, que la independencia de campo es susceptible de ser incrementada mediante un entrenamiento lingüístico perceptivo y en condiciones específicas.

Con relación a la percepción del funcionamiento familiar, la investigación realizada por Palomar y Márquez (1989), titulada "Relación entre la Escolaridad y la Percepción del Funcionamiento Familiar", en la cual participaron 607 padres de familia, confirma que la escolaridad juega un papel central en la percepción del individuo respecto del funcionamiento familiar; las personas entrevistadas tienden a percibir favorablemente algunas de las dimensiones familiares, cuando el padre y/o la madre han alcanzado un nivel escolar alto y, a su vez, tienden a hacerlo más desfavorablemente cuando el padre o la madre sólo alcanzaron niveles medios de escolaridad. Por otra parte, cuando la variable independiente es la escolaridad del entrevistado, su efecto sobre la percepción de algunas dimensiones del funcionamiento de su familia, tiende a ser lineal, es decir, mientras mayor es su escolaridad, será más favorable su percepción.

Por ende, los resultados de estas investigaciones permiten apreciar el importante papel que juega la familia y principalmente los padres en la socialización de sus hijos; sin embargo, considerando que el funcionamiento familiar tiene relación con los recursos económicos y el nivel educativo del grupo familiar (y entre estas últimas existe una fuerte asociación), es viable afirmar que la escolaridad adquirida también modifica las pautas de conducta aprendidas en el hogar, relacionadas con el funcionamiento de la familia.

Los resultados de la investigación de carácter prospectivo realizada por Páramo y Tinajero (1992) titulada "Influencia de la estructura normativa familiar sobre el estilo cognitivo dependencia- independencia de campo", con la participación de 316 niños y niñas en edades comprendidas entre 12 y 14 años procedentes de familias con distinta estructuración normativa, confirman parcialmente las previsiones realizadas desde el modelo de la diferenciación, en el cual aquellos ambientes familiares que posibilitan a los niños la organización y estructuración de sus propias experiencias tienden a favorecer el desarrollo de un estilo independiente de campo, mientras que en las familias rígidas, predomina el estilo dependiente de campo.

Estos resultados de acuerdo con las autoras del estudio, confirman los hallazgos de la investigación realizada por Witkin y 
colaboradores, quienes además encuentran en otro estudio que "dimensiones de interacción familiar, relacionadas con aspectos normativos (nivel de asertividad permitido y grado de restricción en la relación con los demás) pueden actuar en favor o en contra del proceso de diferenciación" (Witkin, 1962, citado en Páramo y Tinajero, 1992, p. 6).

En las últimas décadas, las investigaciones sobre funcionamiento familiar en el campo educativo han cobrado importancia para establecer su relación con el rendimiento académico.

Al respecto, el estudio realizado por Sánchez y Valdés (2011) titulada "Una aproximación a la relación entre el rendimiento académico y la dinámica y estructura familiar en estudiantes de primaria", en la que participaron 80 alumnos provenientes de familias nucleares y 63 monoparentales, con el objetivo de determinar si existían diferencias en la dinámica familiar de los estudiantes y si estos aspectos se relacionaban con el desempeño académico, concluye que el grupo de estudiantes proveniente de familias monoparentales tiene una percepción más negativa de la dinámica familiar, en aspectos relacionados con la autoridad, la comunicación y la valoración de la familia y por otra parte, que el desempeño académico se relaciona con la dinámica, pero no con la composición familiar, lo cual significa que la monoparentalidad por sí misma no tiene relación directa con los logros escolares de los hijos, sino con las afectaciones que surgen de la dinámica propia de las familias.

\section{Pregunta de Investigación y Objetivos}

\section{Pregunta de investigación}

¿Cuáles son los estilos cognitivos y la percepción del funcionamiento familiar de estudiantes de $1^{\circ}$ a $3^{\text {oa }}$ de básica primaria de una Institución Educativa del sector oficial del municipio de Dosquebradas (Risaralda)?

\section{Objetivo General}

Caracterizar los estilos cognitivos en la dimensión dependencia/independencia de campo y la percepción de la función familiar en estudiantes de básica primaria de una Institución educativa del sector oficial del municipio de Dosquebradas.

\section{Objetivos Específicos}

Identificar los estilos cognitivos en la dimensión dependencia/independencia de campo de los estudiantes según el género y el grado escolar.

Analizar la percepción del funcionamiento familiar según el grado escolar de los estudiantes de básica de una Institución educativa del sector oficial del municipio de Dosquebradas.

\section{Descripción Teórica}

El término estilos cognitivos, alude a una expresión de la personalidad consistente con una disposición al uso de ciertas habilidades cognitivas. Para García (1989), el estilo cognitivo es un concepto que refleja el intento de los psicólogos de cubrir el vacío entre los factores de personalidad y la cognición.

Existen varias definiciones de estilos cognitivos, las cuales se diferencian especialmente por el énfasis que hacen en aspectos cognitivos. No obstante, coinciden en que es un modo característico de funcionamiento cognitivo, una actitud en el ámbito de la percepción, que se constituye en un elemento diferenciador de los seres humanos, en una dimensión amplia de la personalidad que se manifiesta de manera estable, profunda y en un alto nivel de generalización.

A pesar de los diversos enfoques y definiciones se ha llegado a establecer que el estilo cognitivo es la forma característica de pensamiento individual que permite percibir, recordar y usar la 
información para resolver un problema de un modo determinado y diferenciado. Enfoque que se comparte como concepto de referencia en el presente estudio en concordancia con Kogan (1971), un autor representativo del estudio de los estilos cognitivos, quien plantea que son variaciones individuales en los modos de percibir, recordar, pensar, o distintas maneras de aprehender, almacenar, transformar y utilizar la información. Por su parte, García (1989, p. 23), define los estilos cognitivos como "una herramienta conceptual sintetizadora, para resumir el método cognitivo básico en que una persona se enfrenta a todas las formas de estimulación sensorial".

Para Grigorenko y Sternberg (1995), los estilos cognitivos permiten la integración de la cognición y la personalidad y están convencidos de que el estudio sobre los estilos puede ayudar a formar una teoría que incluya estos dos aspectos. Estos autores describen tres aproximaciones psicológicas al estudio de los estilos. La primera se centra en la cognición, la segunda, en la personalidad y la tercera, en la actividad. Las dos primeras de estas aproximaciones, corresponden a períodos de un desarrollo activo en el campo de los estilos y la tercera, enfatiza la perspectiva educativa.

Es importante anotar que los estilos son conjuntos de rasgos estables intelectuales, afectivos y emocionales a través de los cuales las personas interactúan en los diversos ambientes de aprendizaje. Los estilos cognitivos, se encuentran integrados por habilidades cognitivas y meta cognitivas y se infieren de las diferencias individuales en la organización, procesamiento de la información y la propia experiencia.

Witkin y otros autores han identificado y estudiado diferentes dimensiones de los estilos cognitivos, entre las cuales se encuentran dependencia/independencia de campo, impulsividad/reflexividad, visualizador/verbalizador, visual/háp- tico (táctil), estilo conceptual o serial/ holístico.

La dimensión Dependencia-Independencia de campo fue identificada por Witkin y Goodenough (1985), como una amplia validez predictiva respecto a la personalidad y a la conducta social del individuo debido a que es el enfoque característico que utiliza la persona ante una gama de situaciones, lo cual abarca actividades perceptuales e intelectuales. Este estilo, en palabras de los autores, se puede definir como:

"el grado en que la persona percibe una parte del campo perceptivo, como separado del contexto que lo rodea, en vez de hacerlo como si estuviera incluido en el, o al grado en que la organización del campo predominante determina la percepción de sus componentes; o por decirlo en palabras corrientes el grado en que la persona percibe de manera analítica”.(Witkin, et al 1985, p. 38).

En este sentido, la dimensión dependencia-independencia de campo, representa una bipolaridad, en la cual en uno de los extremos se ubica la dimensión Independencia de campo perceptivo (I.C.) la cual, mirada desde el punto de vista operativo, viene a ser la alta aptitud para reestructurar un campo perceptivo complejo y en el otro polo se ubica la dimensión dependencia de campo perceptivo (D.C.), entendida como la baja aptitud para la reestructuración de un campo perceptivo complejo. Los sujetos independientes de campo (IC) son los que tienden a percibir la información de manera analítica y sin dejarse guiar por el contexto y, por el contrario, aquellos que tienden a percibir de manera más global, son dependientes de campo (DC). Es de aclarar que la dimensión dependenciaindependencia de campo-DIC- es una dimensión continua que expresa la mayor o menor capacidad de un sujeto para reestructurar campos complejos. 
La bipolaridad dependencia-independencia de campo como dimensión cognitiva, tiene un substrato biológico, importante desde el punto de vista psicológico y pedagógico, porque de él depende el grado de modificabilidad o educabilidad de los estilos, sin olvidar los factores de interacción social que influyen en la dimensión cognitiva, más aún cuando el propio Witkin (citado por García, 1989) afirma que "Ios factores de socialización son de una importancia irrefutable en el desarrollo de las diferencias individuales en la D.I.C" (p. 10), y en consecuencia, no es posible hablar de determinación biológica en la dimensión dependencia-independencia de campo, sino de la interacción entre los factores biológicos, sociales, culturales y familiares (Hederich, et al, 1995).

En la actualidad, ambos estilos son igualmente valorados, considerando que diferentes circunstancias o tareas solicitan más de un estilo que del otro. En este caso "el efecto del estilo sobre el desempeño de un individuo es positivo o negativo dependiendo de la naturaleza de la tarea".
(Hederich, 2001, p. 14). En la Tabla 1, se presentan algunas características de los estilos en la dimensión dependenciaindependencia de campo, en relación con el aprendizaje.

Con relación a los factores familiares, numerosos estudios han destacado el importante papel de la familia en los procesos de aprendizaje y desarrollo de los hijos. Aspectos relacionados con la estructura familiar (composición del grupo), las características de los padres (coercitivos, sumisos, perfeccionistas, etcétera), el tipo de comunicación al interior de la familia (clara, directa y congruente), los métodos disciplinarios utilizados (incongruente, restrictiva y hostil), así como las relaciones de los padres, se vinculan con problemas de conducta escolar en los adolescentes (Mejía, et al 1989; Demo y Acock, 1996)

Si se tiene en cuenta que la familia es la unidad básica y más importante de un Estado, en el proceso de socialización se puede considerar que los hijos estarán

\section{Tabla 1. Características de los estilos en la dimensión dependencia e independencia de campo en relación con el aprendizaje}

\begin{tabular}{|l|l|}
\hline Estilo Dependiente de Campo & Estilo Independiente de Campo \\
\hline Perciben globalmente. & Perciben analíticamente. \\
\hline Son mejores para aprender material de contenido social. & $\begin{array}{l}\text { Necesitan ayuda para concentrarse en el material de } \\
\text { contenido social. }\end{array}$ \\
\hline Memorizan mejor información social. & $\begin{array}{l}\text { Se les debe enseñar a usar el contexto para manejar } \\
\text { información social. }\end{array}$ \\
\hline Necesitan instrucciones para llevar a cabo una tarea. & $\begin{array}{l}\text { Son capaces de resolver problemas y llevar a cabo } \\
\text { tareas sin necesidad de instrucciones. }\end{array}$ \\
\hline $\begin{array}{l}\text { Suelen aceptar la organización que se les impone y son incapaces } \\
\text { de reorganizar. }\end{array}$ & Pueden analizar una situación y reorganizarla. \\
\hline $\begin{array}{l}\text { Están acostumbrados a que les digan qué hacer. Necesitan } \\
\text { retroalimentación. }\end{array}$ & $\begin{array}{l}\text { Tienen iniciativa y pueden utilizar sus propios criterios } \\
\text { para desarrollar tareas. No necesitan retroalimentación. }\end{array}$ \\
\hline Son sensibles a la crítica. & Las críticas no los afectan mucho. \\
\hline Requieren estructuras, metas y refuerzos externos para trabajar. & Suelen tener metas y refuerzos internos. \\
\hline $\begin{array}{l}\text { Carecen de un sentido definido de autonomía. Necesitan que se } \\
\text { les enseñe a usar ayudas mnemónicas. }\end{array}$ & $\begin{array}{l}\text { Tienen un sentido definido de autonomía. Pueden usar } \\
\text { sus propias estructuras en situaciones no estructuradas. }\end{array}$ \\
\hline Les gusta trabajar con otros. Son fácilmente influenciados por otros. & Les gusta trabajar solos. Son difícilmente influenciables. \\
\hline
\end{tabular}

Fuente: Adaptado de Alonso, Gallego y Honey (1995); Almaguer (1998) y Lozano (2000). 
orientados a reproducir ciertas pautas de funcionalidad o disfuncionalidad semejantes a las que viven en sus hogares; ello, aunado al impacto que otros agentes de socialización (escuela, cultos religiosos, grupos de pares, medios de comunicación), puedan generar en sus patrones de vida familiar.

La funcionalidad familiar es un concepto de carácter sistémico, con características relativamente estables que permean las relaciones de los sujetos que la conforman.

"Es el conjunto de relaciones interpersonales que se generan al interior de cada familia y le confieren identidad propia. La dinámica relacional conforma la calidad del ambiente $y$ la atmósfera del hogar, le imprime al mismo un clima agradable, de satisfacción o un clima desagradable, de tensión y regula el desarrollo del grupo familiar como un todo"(Castellón y Ledesma, 2012, p. 1).

Diferentes estudios realizados (Beavers y Hampson, 1995; Moos, Moos y Trickett, 1989; Olson, 1991, (citados por González, Gimeno, Meléndez, y Córdoba, 2012), plantean que la funcionalidad del sistema familiar está asociada a diferentes características entre las cuales se destacan, los niveles de cohesión, flexibilidad, comunicación, estrés, conflictos, emociones y vínculos.

Un funcionamiento familiar sano, posibilita cumplir exitosamente con los objetivos y funciones que histórica y socialmente se le han asignado a la familia entre ellas, la función biosocial, económica, cultural y afectiva y la función educativa. Estas funciones se relacionan con la satisfacción de necesidades afectivo-emocionales y materiales, la transmisión de valores éticos y culturales, la promoción y condición del proceso de socialización, el establecimiento y mantenimiento del equilibrio, el establecimiento de patrones para las relaciones interpersonales (educar para la convivencia social), la creación de condiciones propicias para el desarrollo de la identidad personal y la adquisición de la identidad sexual(Ares, 2004).

En coherencia con lo anterior, un funcionamiento familiar saludable, exige el desarrollo de una dinámica familiar adecuada, comprendida como "el establecimiento de determinadas pautas de interrelación en el grupo familiar, mediadas o matizadas por la expresión de sentimientos, afectos y emociones de los miembros entre sí, y en relación con el grupo en su conjunto"(Hernández, 2005, citado en Zicavo, Palma y Garrido, 2012, p. 221).

De acuerdo con ello, un buen funcionamiento familiar incluye características como la adaptabilidad o flexibilidad, la cohesión, el clima emocional, la comunicación efectiva, un buen afrontamiento y la resolución de problemas. Una familia que cumple adecuadamente sus funciones de protección y cuidado, fomenta el desarrollo de competencias en sus miembros, lo que contribuye a prevenir o enfrentar problemáticas de salud.

\section{Metodología}

Estudio descriptivo de corte transversal, realizado con el objetivo de caracterizar los estilos cognitivos y la percepción del funcionamiento familiar en estudiantes de básica, de una institución educativa del sector oficial. El diseño de la investigación es no experimental, por cuanto se observan las variables y sus posibles relaciones en su contexto natural, partiendo del hecho que ya ocurrieron o se dieron en la realidad sin intervención directa del investigador.

La población la conformaron 251 estudiantes de 6 a 10 años de $1^{\circ}$ a $3^{\circ}$ grado de educación básica de una Institución Educativa del municipio de Dosquebradas. La muestra la conformaron 69 estudiantes, seleccionada mediante la técnica de muestreo simple aleatorio.

Para la caracterización de los estilos cognitivos de los niños y las niñas se utilizó el Instrumento denominado Test de figu- 
ras enmascaradas para niños (Children's Embedded Figures Test: CEFT). Esta prueba es una adaptación del test de figuras enmascaradas (Embedded Figures Test: EFT) y es empleada para evaluar la dimensión dependencia-independencia de campo, realizada por Karp y Konstadt en 1963.

Para la valoración de la percepción de la función familiar se utilizó la escala APGAR Familiar,diseñada por Smilkstein (1978) y revisada posteriormente por Smilkstein, Ashworth y Montano (1982). Es un instrumento útil para evidenciar la forma en que una persona percibe el funcionamiento de su familia en un momento determinado.

El instrumento empleado en el estudio, es tomado del Servicio Andaluz de SaludEspaña (Junta de Andalucía, 2002; Bellón, Delgado, Luna \& Lardelli, 1996). Se trata de un cuestionario que puede ser auto administrado o hetero administrado y consta de 5 ítems, cuyo puntaje total fue evaluado como: normo funcional: $12-15$ puntos, disfuncional leve: 8-11 puntos y disfuncional grave: 5-7 puntos.

\section{Resultados}

\section{Características de los estilos cognitivos de los estudiantes}

En el estudio participaron 69 estudiantes de $1^{\circ}$ a $3^{\circ}$ grado de básica primaria, de los cuales el $62 \%$, corresponde al género masculino, y el $38 \% \%$, al género femenino, lo cual equivale a 26 niñas de la muestra.

Los estilos cognitivos se refieren a las formas o modos de percibir, recordar, organizar, procesar, pensar y resolver problemas. No se trata de habilidades, sino más bien, de la manera preferida como se usan las habilidades (Sternberg y Grigorenko, 1997). Los estilos cognitivos de los niños y las niñas identificados en la muestra de estudio, se muestran en la siguiente gráfica:

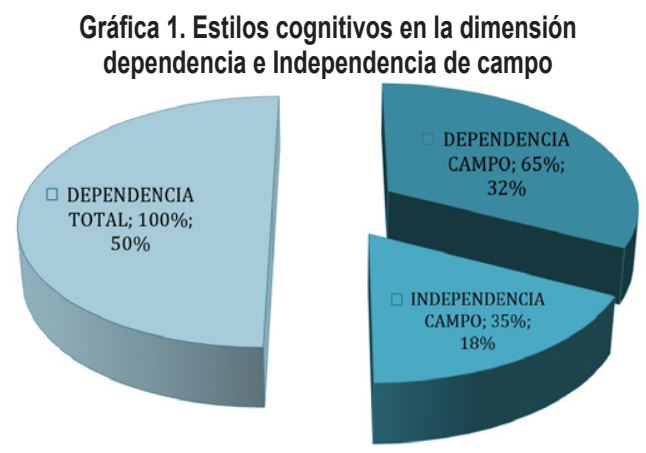

El $65 \%$ de los estudiantes tienen un estilo cognitivo Dependiente de Campo, mientras el $35 \%$ es Independiente de Campo, lo que demuestra que el género no es directamente proporcional al estilo cognitivo. Debe anotarse que aun cuando el porcentaje de hombres dependientes de campo supera en más de la mitad a los independientes de campo, Witkin (1974) plantea que las diferencias entre sexos son leves, si se les compara con las amplias diferencias entre individuos del mismo sexo, ya que la tendencia es que la mujer se incline hacia la dependencia de campo y el hombre a la independencia de campo.

De acuerdo con el género, los estilos cognitivos en la dimensión dependencia e independencia de campo identificados, confirman una mayor inclinación de los niños a la independencia de campo, con relación a las niñas, como se evidencia en la gráfica 2 .

La gráfica 2, indica que el $42 \%$ del total de la muestra de los niños son dependientes de campo, mientras que el $20 \%$ es independiente de campo. Por el contrario, en las niñas el $23 \%$ del total es dependiente de campo y el $14 \%$ es independiente de campo.

Varios investigadores entre ellos Kimura (1992); Buxo (1991) citados en García (1989) señalan que los hombres tienden a ser más independientes de campo que las mujeres. Si se analiza desde el punto de vista biológico, el argumento tiene su justificación en el nivel de diferenciación 
Gráfica 2.

Género en relación con el estilo cognitivo

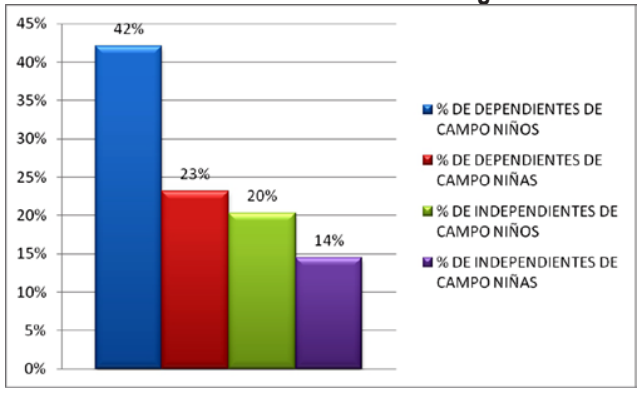

neurológica y en la capacidad de reestructuración cognitiva que ocurrió por causa de la evolución de la especie, a través de la cual, los hombres se dedicaron a las tareas de la caza, lo cual implicaba mayor habilidad viso-espacial, en tanto las mujeres se dedicaron a las actividades que implicaban motricidad fina y desarrollo del lenguaje para cuidar los hijos.

En lo que respecta al estilo cognitivo, según el grado escolar, los datos obtenidos muestran, que a medida en que se "asciende" en los distintos grados de escolaridad, la capacidad de reestructuración se mantiene estable y existe una mayor tendencia de los estudiantes, a la independencia de campo, como se aprecia en las siguientes gráficas.

Gráfica 3. Estilos cognitivos en la dimensión dependencia-independencia de campo en estudiantes de primer grado

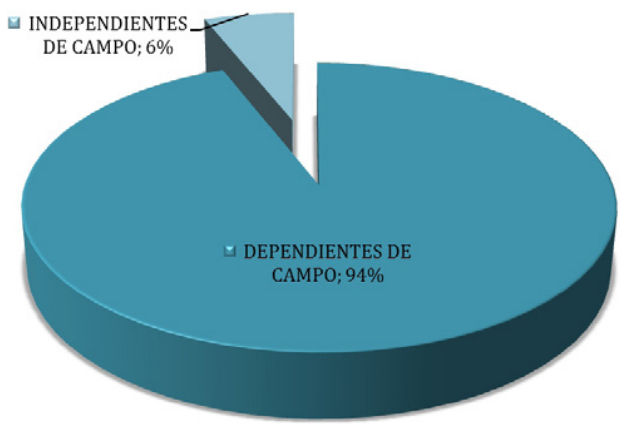

En edades comprendidas entre los 7 y 8 años existe una mayor tendencia hacia la dependencia de campo, la cual disminuye entre los 9 y 12 años, momento en el que se desarrolla la independencia de campo. De acuerdo con los resultados del estudio, la mayoría de los niños y las niñas de primer grado, son dependientes de campo en una proporción del $94 \%$.

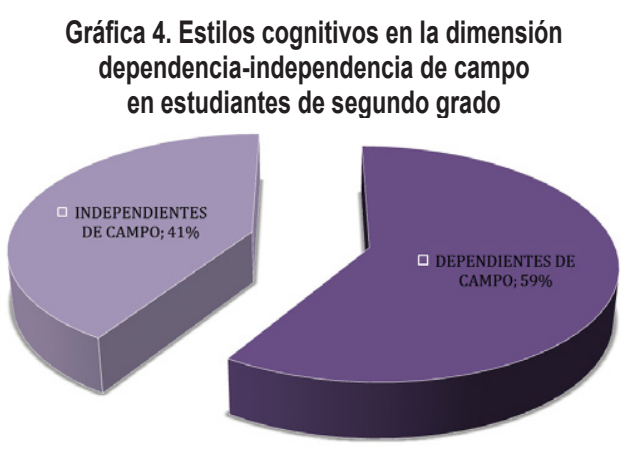

La gráfica 4, indica que en segundo grado, la proporción de niños y niñas dependientes de campo es del $59 \%$, respecto del $41 \%$, que son independientes de campo. Es importante señalar que en la medida que los niños y las niñas crecen y maduran se exponen a niveles escolares más complejos que les permiten desarrollar un pensamiento formal y la independencia de campo (García, 1989).

\section{Gráfica 5. Estilos cognitivos en la dimensión dependencia-independencia de campo en estudiantes de tercer grado}

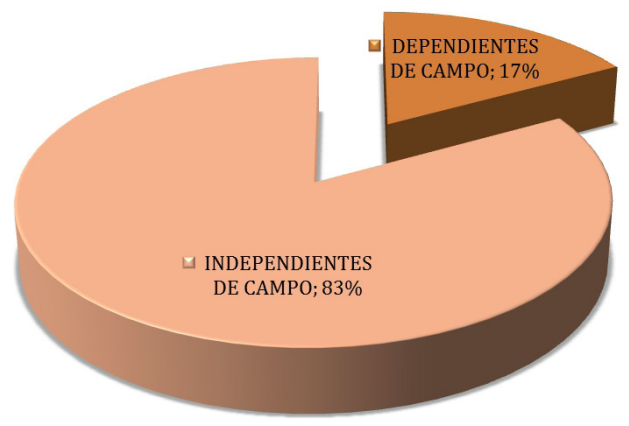

La gráfica muestra, que en grado tercero, los niños y las niñas, presentan un mayor porcentaje de independencia de campo representado en un $83 \%$, con relación a los estudiantes dependientes 
de campo que están representados por el $17 \%$. Como ya se ha afirmado, se puede apreciar un claro aumento hacia la independencia de campo a medida que aumenta la edad. Este aumento parece ser modulado por el desarrollo evolutivo mismo. Dicho aumento es relativamente homogéneo, al mantenerse las diferencias interindividuales a través de los años.

La edad escolar parece ser un factor que conlleva a una reestructuración cognitiva apreciable; las personas tienden a ser más independientes de campo, con el incremento de la edad. Para autores como Amador (1992), la reestructuración cognitiva, el procesamiento de la información y la eficiencia se van perfeccionando con la edad biológica. Es por ello, que los estudiantes independientes de campo, puesto que presentan mayores habilidades visuoespaciales y de reestructuración perceptiva, serán más eficaces que los estudiantes dependientes de campo en tareas de procesamiento simultáneo (memoria espacial y visualización de cubos), que utilizan material visoespacial.

\section{Caracterización de la percepción del funcionamiento familiar}

La percepción del funcionamiento familiar, manifiesta el grado de satisfacción de los integrantes de la familia en las dimensiones básicas, relacionadas con la forma como el grupo familiar es capaz de enfrentar las crisis y resolver los problemas (adaptación), valorar la forma en que se permiten las expresiones emocionales (afecto), el crecimiento individual, la interacción sobre la base del respeto y la autonomía, así como los recursos de los cuales se dispone para atender las necesidades.

En este sentido, un buen funcionamiento se asocia con la capacidad de la familia para responder a las demandas de su entorno, en tanto la disfunción familiar puede obedecer a la falta de interacción dentro y fuera del grupo familiar, lo cual se refleja en una inadecuada comunicación y relaciones conflictivas con las personas y los grupos.

Los resultados obtenidos de la aplicación de la prueba APGAR familiar se ilustran en la siguiente tabla:

Tabla 2.

\section{Percepción de la función familiar}

\begin{tabular}{|l|c|c|}
\hline \multicolumn{1}{|c|}{ Tipo de percepción } & $\begin{array}{c}\text { Número de } \\
\text { Estudiantes }\end{array}$ & Porcentaje \\
\hline Buen funcionamiento (BF) & 23 & $33 \%$ \\
\hline Disfuncional Leve (DL) & 43 & $62 \%$ \\
\hline Disfuncional Grave (DG) & 3 & $5 \%$ \\
\hline Total & 69 & $100 \%$ \\
\hline
\end{tabular}

Fuente. Elaboración propia

De acuerdo con los datos presentados en la tabla anterior, la valoración de la percepción del funcionamiento familiar, indica que el $33 \%$ de los estudiantes percibe buen funcionamiento en el entorno familiar, es decir que el contexto familiar se caracteriza por una funcionalidad sociofamiliar estable, mientras que un $62 \%$, corresponde a Disfuncional leve y un $5 \%$ lo caracteriza como Disfuncional Grave.

Según el grado escolar, el $62 \%$ de los estudiantes de primer grado perciben una disfuncionalidad leve, mientras que el $35 \%$ perciben un buen funcionamiento familiar y el 3\%, restante lo perciben como disfuncional grave.

La disfuncionalidad leve continua siendo una variable con un índice de frecuencia alto en cuanto a la percepción del funcionamiento familiar de los estudiantes de segundo grado de la Institución Educativa, representada en un 53\%, frente a un $35 \%$ que percibe un buen funcionamiento familiar.

En el grado tercero, los estudiantes perciben una disfuncionalidad leve, en un $72 \%$, que corresponde al porcentaje más elevado en relación con los dos grados anteriores, mientras que la percepción 
de un buen funcionamiento familiar es representada en un $28 \%$.

La funcionalidad familiar está asociada a las posibilidades de las familias y éstas a su vez, con la capacidad adaptativa y los recursos que han adquirido durante el ciclo vital. En este sentido, un buen funcionamiento (normofuncional) percibido por el $33 \%$ de los estudiantes, indica que el clima familiar se mantiene estable; las normas de funcionamiento al interior de la familia, regulan las relaciones entre sus integrantes para conservar el equilibrio.

La disfuncionalidad del sistema familiar, es percibida por el $62 \%$ de los estudiantes como leve y en un $5 \%$ grave. Esta situación, puede conceptualizarse como el mantenimiento de un deficiente funcionamiento de la familia a través del tiempo y un quebrantamiento de las funciones culturalmente establecidas. Toda familia otorga a sus integrantes elementos propicios para su autorrealización, pero también de manera contradictoria puede desarrollar roles que produzcan conductas inadecuadas creando un clima, que de mantenerse en el tiempo, puede convertir a una familia funcional en disfuncional, y en consecuencia, no contribuir al desarrollo sano de sus integrantes (Reynalte, 2010).

Varios estudios, entre otros, realizados por Sánchez, Camacho, Valencia \& Rodríguez (2008); Hernández, Cargill y Gutiérrez (2012), han demostrado que la disfuncionalidad familiar es un factor importante que condiciona la aparición de problemas socioculturales y de salud, como fármaco-dependencia, embarazo, enfermedades de transmisión sexual, deserción escolar, depresión, suicidio.

\section{Discusión y conclusiones}

El propósito del estudio se orientó a la caracterización de los estilos cognitivos y la percepción del funcionamiento familiar de los estudiantes de primer a tercer grado de básica de una institución educativa.
La caracterización de los estilos cognitivos en relación con el género de los estudiantes que participaron en el estudio, confirma el hecho de una mayor tendencia del género masculino a la independencia de campo, mientras que la dependencia de campo tiende a ser característica del género femenino. Witkin y sus colaboradores (1962), refieren que en las culturas occidentales los varones tienden a presentar mayor tendencia a la independencia de campo que las mujeres. Hederich et al (1995), consideran que el desarrollo del estilo cognitivo depende de factores de carácter biológico presentes en cada uno de los sexos, relacionados con factores de orden cultural siempre presentes en la diferenciación entre los géneros. Entre los factores de orden cultural se encuentran los estilos de crianza, el modelamiento por parte de la familia, la autoridad y la autonomía propias de cada grupo, en particular.

Por otra parte, los resultados del estudio confirman que existe correspondencia entre la edad, el grado escolar y la predisposición hacia la independencia de campo. En este sentido, se pudo observar un incremento del estilo independiente de campo en la medida en que los estudiantes avanzan en su proceso escolar, lo que es acorde con lo planteado por Witkin et al, citados en Amador (1992) cuando señalan que a mayor tiempo de permanencia en la escolaridad, mayor será la tendencia hacia la independencia al medio, lo que a su vez indica que existe una relación directa entre el grado escolar, la edad y la capacidad de reestructuración cognitiva de los estudiantes.

Durante los primeros años de vida los niños y las niñas, requieren el apoyo externo para comprender la realidad del mundo constituida por símbolos y significados culturales a través del lenguaje, la mediación y la actividad humana. En este momento el pensamiento en la niñez es intersubjetivo, sensible a las claves externas o dependiente de campo gracias a que su aprendizaje y comprensión requieren de la explicación del otro. Años 
más tarde, desarrollan una capacidad de pensamiento formal que les permite manejar de manera analítica y autónoma la información disponible, siendo un sujeto intrasubjetivo con un estilo independiente de campo.

El estilo cognitivo depende en mayor parte de la interacción familiar y la escuela se convierte en un escenario que instruye y permite dar uso a las herramientas cognitivas que el niño y la niña desarrollan. De acuerdo con Hederich, et al (1995), "los mecanismos de reproducción sociocultural (y cognitiva) de los grupos humanos tienen su expresión más directa en las prácticas de crianza de los hijos. A su vez estas prácticas influyen de forma directa en la constitución del estilo cognitivo de cada persona" (p. 62).

Bronfenbrenner (1987) considera que la familia es el sistema que define y configura en mayor medida el desarrollo de la persona desde su concepción. Para el autor, el entorno es algo que trasciende la situación inmediata y afecta directamente a la persona en desarrollo; por ello, es de suma importancia la relación que se establece dentro y fuera de la familia, pues permite la instauración de un proceso de desarrollo al interior de contextos como la escuela, la familia y la sociedad en general. Este desarrollo es importante porque allí se crean los primeros vínculos afectivos, que repercuten en la conducta del individuo a lo largo del ciclo vital.

La familia es un conjunto organizado e interdependiente de personas en constante interacción, regulado por reglas y funciones dinámicas tanto internas como externas (Minuchin, 1986, Andolfi, 1993; Musitu et al., 1994, Rodrigo y Palacios, 1998, citados en Espinal, Gimeno y Gonzáles, 2006).Se considera que una familia sana o funcional presenta características relacionadas con la comunicación clara y directa, la definición de roles, la autonomía de los integrantes, la cohesión y solidaridad entre los integrantes de la familia y la habilidad para resolver problemas
(Ponzetti y Long, 1989; Mansour y Soni, 1986, citados en Guadarrama, Márquez, Veytia y León 2011).

La percepción de la función familiar, referida a la aprehensión de la realidad familiar por parte de las personas que la integran, indica que un $33 \%$ de los estudiantes perciben buen funcionamiento familiar, seguido de un $66 \%$ asociado a una disfuncionalidad leve y un $5 \%$ como disfuncionalidad grave.

Los resultados obtenidos de la percepción del funcionamiento familiar, según el grado escolar, indican que un alto porcentaje de los estudiantes percibe una disfuncionalidad leve que va en aumento de primer a tercer grado (62\% al $72 \%$ ), lo cual puede explicarse por las características de la muestra poblacional ubicada en estratos 1 y 2 , en donde los problemas sociales, económicos y culturales, entre otros, afectan el entorno y la dinámica familiar.

Al respecto, De la Revilla y Fleitas (1994), plantean que la familia como microsistema, es influenciado por otros sistemas y se rige por las normas que éstos le asignan dependiendo de la cultura y la época. Por lo tanto, no es posible hablar de un modelo de familia normal abstracto y universal, sino de una serie de funciones que la familia debe ser capaz de cumplir para garantizar el sano desarrollo de los hijos.

En esta perspectiva, el funcionamiento familiar comprende una gran diversidad de factores relacionados con el contexto social y cultural. Smilsktein, et al, citados por De la Revilla y Fleitas (1994) señalan que el sistema familiar elabora respuestas adaptativas frente a las influencias del entorno, representado por acontecimientos vitales estresantes y sus implicaciones en el medio interno, así como las transiciones que tienen lugar en el ciclo vital. No obstante, este equilibrio puede verse amenazado por el desencadenamiento de acontecimientos vitales, que conllevan a una crisis familiar, situación que puede 
considerarse necesaria para el crecimiento y evolución del sistema familiar.

En caso de crisis, el sistema familiar acude a sus recursos internos y externos si dispone de los mismos, para producir una respuesta adaptativa que contrarreste los efectivos negativos o, elabora nuevas respuestas modificando su funcionamiento interno, alcanzando un nuevo equilibrio diferente al anterior. También, puede ocurrir que el sistema no disponga de los recursos y por tanto, no pueda resolver la crisis. En esta situación, el equilibrio se rompe y entra en una situación de disfunción familiar, la cual puede ser superada aportando los recursos necesarios, Io cual no siempre es posible o bien, si la disfunción permanece puede conducir a la ruptura y disolución del sistema familiar (Smilkstein, 1978).

Para concluir, es preciso indicar que los permanentes cambios en nuestro entorno social, económico, tecnológico e ideológico, acentuados por fenómenos como la globalización, están demandando a las familias, cambios sustanciales en su composición y en su organización interna, que generan desorientación y estrés, para los cuales las familias no siempre tienen la posibilidad de dar respuesta, ni la suficiente adaptación, situación que conduce a conflictos y crisis que pueden desencadenar angustia, patología y la ruptura (Espinal, Gimeno, \& González, 2006).

Es necesario crear espacios con la comunidad educativa que promuevan la reflexión en torno a la función familiar, desde un enfoque psicopedagógico, que destaque la importancia del entorno familiar, que en la mayoría de los casos de la muestra estudiada presenta disfuncionalidad leve. Estas reflexiones primero deben hacerse para sí mismo; meditar sobre la propia historia, hábitos, procedencia familiar, cultura, gustos, para así comprender los procesos de desarrollo del estudiante en sus distintas dimensiones y el papel de la familia en torno a los procesos de formación.

Por otra parte, es probable que la percepción de la funcionalidad familiar ejerza influencia en la autoestima y la seguridad de los niños y las niñas. Conocer esta posible influencia, permitirá realizar estudios de mayor profundidad que establezcan la forma más adecuada que debe asumir la función familiar, así como su incidencia en la configuración y/o adopción de un determinado estilo cognitivo, teniendo en cuenta que los estilos no determinan el potencial para aprender, sino que determinan más bien, la forma como se lleva a cabo el aprendizaje (Hederich, 2004).

\section{Bibliografía}

Alegre Palomino, $Y$ \& Suárez Bustamante, $M$. (2006). Instrumentos de Atención a la Familia: El Familiograma y el APGAR familiar. En revista Rampa, 1(1):48 - 57

Alonso, C, Gallego, D \& Honey, P. (1995). Los estilos de aprendizaje. Procedimientos de diagnóstico y mejora. Bilbao: Ediciones Mensajero.

Almaguer, T. (1998). El desarrollo del alumno: características y estilos de aprendizaje. México: Trillas.

Amador, J A. (1992). Eficiencia en la resolución de tareas de aprendizaje en función del estilo cognitivo dependencia-independencia de campo. Tesis Doctoral, Facultad de Psicología,Universidad de Barcelona.

Ares, P. (2004). Psicología de Familia. Una aproximación a su estudio. La Habana: Editorial Félix Varela.

Avanzini, G. (1995). El fracaso escolar. Barcelona: Herder.

Bellón, J. A. Delgado, A. Luna del Castillo, J. D. Lardelli, P. (1996).Validez y fiabilidad del cuestionario de función Familiar APGARfamiliar. Atención primaria; 18: 289-96. Disponible en: http://www.elsevier.es/en/ node/2001226. Recuperado en Marzo 23 de 2013. 
Bronfennbrenner,Urie.(1987). La Ecología del Desarrollo Humano. Barcelona: Paidós.

Carretero, M \& Palacios, J. (1982). "Los estilos cognitivos: Introducción al problema de las diferencias cognitivas individuales". Infancia y Aprendizaje, No. 17, pp. 20-28.

Castellón Castillo, S \& Ledesma Alonso, E. (2012). El funcionamiento familiar y su relación con la socialización infantil. Proyecciones para su estudio en una comunidad suburbana de Sancti Spíritus. Cuba. En Contribuciones a las Ciencias Sociales.

De la Revilla A. L, Fleitas C.L. (1994). Función y disfunción familiar. En: De la Revilla, A. L. Conceptos e Instrumentos de la atención familiar. Barcelona. España. Doyma.

Demo, D. H \& Acock, A.C. (1996) Family structure, family process and adolescent will-being. En Journal of Research en adolescence. Vol. 6 Nro. 4. pp. 457-488.

Espinal, I., Gimeno, A., \& González, F. (2006). El enfoque sistémico en los estudios sobre la familia. Universidad Autónoma de Santo Domingo. Facultad de Psicología http:// www. uv. es/jugar/EnfoqueSistemico. pdf.

García Ramos, J. (1989). Los estilos cognitivos y su medida: estudios sobre la dimensión dependencia-independencia de campo.Madrid: Centro de Publicaciones del Ministerio deEducación y Ciencia CIDE. pp. 21, 60, 61.

González Sala, F, Gimeno Collado, A, Meléndez Moral, J, Córdoba Iniesta, A. (2012). La percepción de la funcionalidad familiar. Confirmación de su estructura bifactorial. Escritos de Psicología - Psychological Writings [en linea]. Disponible en:<http://www. redalyc. org/articulo.oa? id=271024366005> ISSN 1138-2635

Grigorenko, Elena L \& Sternberg, Robert. J. (1995). Thinking styles. En Saklofske, D. M., y Zeidner, M. (Ed.), International Handbook of Personality and Intelligence (pp. 205229). Nueva York: Plenum Press.

Guadarrama, R, Márquez, O, Veytia, M \& León, A. (2011). Funcionamiento familiar en estudiantes de nivel superior. Revista Electrónica de Psicología Iztacala, 14(2), 179-92.

Hederich, Christian, Camargo, Á, Guzmán Rodríguez, L. y Pacheco Giraldo, J. (1995). Regiones cognitivas en Colombia. Santafé de Bogotá: Universidad Pedagógica Nacional - Centro de Investigaciones- CIUP.
Hederich Martínez, C \& Camargo Uribe, Á. (2001). Estilos Cognitivos en el Contexto Escolar. (Primera edición). Universidad Pedagógica Nacional. Bogotá: IDEP.

Hederich Martínez, C. (2004). Estilo cognitivo en la dimensión dependencia-independencia de campo: influencias culturales e implicaciones para la educación. Tesis Doctoral. Bogotá: Fondo Editorial. Universidad Pedagógica Nacional. pp. 259-268.

Hernández Castillo, L, Cargill Foster, NR. \&Gutiérrez Hernández, G. (2012). Funcionalidad familiar y conducta de riesgo en estudiantes de nivel medio superior Jonuta.Salud en tabasco Vol. 18, No. 1, pp. 14-23.

León Sánchez, D, Camacho Delgado, R, Valencia Ortiz, M., \& Rodríguez Orozco, Alain. R. (2008). Percepción de la función de sus familias por adolescentes de la enseñanza media superior. Revista Cubana de Pediatría, 80 (3), 0-0.

Loscos Lucero, M. (2001). Autorregulación del estilo cognitivo a través del lenguaje. Tesis doctoral. Universidad complutense de Madrid. Recuperado en Mayo 23 de 2013 de: http://biblioteca.ucm.es/tesis/edu/ ucm-t25564.PDF.

Lozano, A (2000).Estilos de Aprendizaje y Enseñanza. Un panorama de la estilística educativa. ITESM Universidad Virtual ILCE. México: Trillas.

Karp, S. A. \& Konstadt, N. (1963). Manual for the Children's Embedded Figures Test. Brooklyn: Authors.(Traducción española de L. Manning: Test de Figuras Enmascaradas para Niños, TEA, Madrid, 1982).

Kogan, N. (1971). Educational Implication of Cognitive Style.Psychology and Educational. Pr'ztice, Foresmin, Illinois.

Mejía, S.P. et al. (1989). Ambiente familiar y problemas de conducta escolar. En revista de Psicología de el Salvador. Vol. VIII. Nro. 31. pp. 89-100.

Mori, Saavedra P. (2002). Personalidad, autoconcepto y percepción del compromiso parental: sus relaciones con el rendimiento académico en alumnos del sexto grado. Tesis Doctoral. Universidad Nacional Mayor de San Marcos. Facultad de Psicología. Escuela de Post-Grado, Lima, Perú.

Palomar Lever, J \& Márquez Jiménez, A. (1989). Relación entre la escolaridad y la 
percepción del funcionamiento familiar.Revista Mexicana de Investigación Educativa. Vol. 4, núm. 8, pp.299-343.

Páramo, María F \& Tinajero, C. (1992). Influencia de la estructura normativa familiar sobre el estilo cognitivo dependencia-independencia de campo: un estudio prospectivo. Revista Infancia y Aprendizaje. Vol. 57, 89-98.

Reynalte, A. (2010). La familia disfuncional. Lectura 5. Capítulo II. Disponible en: http://disfuncionalidadfamiliar-v.blogspot.com/2010/11/lectura-5-capitulo-ii.html.

Sánchez, León, D, Camacho Delgado, R, Valencia Ortiz, M, \& Rodríguez-Orozco, Alain R. (2008). Percepción de la función de sus familias por adolescentes de la enseñanza media superior. Revista Cubana de Pediatría, 80(3) Recuperado en 25 de octubre de 2016, de http://scielo.sld.cu/ scielo.php?script=sci_arttext\&pid=S0034$75312008000300004 \&$ Ing=es\&tlng=es.

Servicio Andaluz de Salud. Consejería de Salud. Percepción de la función familiar APGAR. Disponible en http://www.hvn.es/ enfermeria/ficheros/cuestionario_apgar_familiar.pdf.

Smilkstein, G. (1978). The family APGAR: a proposal for a family function test and its use by physicans. J Fam Pract; 6: 1231-239.

Smilkstein, G; Ashworth, C; Montano, D. (1982). Validez y fiabilidad del APGAR fa- miliar como una prueba de la función de la familia. En Journal of Family Practice, Vol 15 (2), 303-311.

Sternberg, Robert. J \& Grigorenko, Elena L. (1997): Are cognitive styles still y style? American Psychologist, 52 (7), 700712.

Valdés Cuervo, Án, \& Urías Murrieta, M. (2010). Creencias de padres y madres acerca de la participación en la educación de sus hijos. Perfiles educativos, 33(134), 99-114.

Valdés, Á; Esquivel, L \& Artiles K. (2007). Familia y desarrollo. Estrategias de intervención en terapia familiar. México: Manual Moderno.

Witkin, H. A. (1976). Cognitive style in academic performance and in teacher-student relations. En Messick, S. (Ed.), Individuality in learning: Implications of cognitive style and creativity for human development(pp. 38-72). San Francisco: Jossey Bass.

Witkin, H. A. \& Goodenough, Donald R. (1985). Estilos cognitivos. Naturaleza y origen. Madrid: Ediciones Pirámide, S.A.

Zicavo, N: Palma, C \& Garrido, G. (2012). Adaptación y validación del Faces-20- ESP: Re-conociendo el funcionamiento familiar en Chillán, Chile. Revista Latinoamericana de Ciencias Sociales, Niñez y Juventud, 10 (1), pp. 219-234 